\title{
COMMISSION 26 (DOUBLE STARS)
}

\author{
President: Prof. E. Hertzsprung. \\ SECRETARY: Dr G. P. KuIPER.
}

The President pointed out that since several members of the Commission were absent no resolutions would be proposed. He drew attention to the great activity in the field of double stars of the Union Observatory, and invited its Director, Dr Wood, who was present at the meeting, to give an account of the recent work by van den Bos and Finsen. Dr Wood said that the Johannesburg double-star survey was now finished, so that more time had become available for other programmes such as that of the systematic measurement of known pairs, and of the examination for duplicity of stars of large proper motion.

Dr Luyten reported on the results of his Bruce survey of proper motions in the southern sky, which was now completed. Nearly Iooo pairs were found with common proper motion exceeding about $0^{n} \cdot 05$ per annum.

Dr Kuiper made some remarks on the orbit of the short-period binary BD- $8^{\circ} 4352$, found by him in 1934 . The period is about $I \cdot 72$ years, the apparent orbit nearly circular. He also mentioned that most of the stars of large known parallax north of $-20^{\circ}$ had been examined for duplicity, but that much remained still to be done for stars for which only the proper motions are known to be large.

Dr Hertzsprung stated that the 180 plates with double-star photographs obtained by him in the fall of 1937 with the 36 -inch refractor of the Lick Observatory had all been measured. The results showed the usual satisfactory accuracy, the mean error of each co-ordinate being $\pm 0^{\prime \prime} \cdot$ or per plate.

There was no further discussion. The Draft Report was unanimously adopted.

\section{COMMISSION 27 (VARIABLE STARS)}

Acting President: Mr H. Grouilier.

SECRETARY: Dr FÉLIX DE ROY.

La Commission rend un hommage ému à la mémoire de son ancien Président, feu le Prof. Nijland, décédé depuis la dernière Assemblée Générale. Le Président de séance rappelle que Nijland appartenait à ce groupe de pionniers que l'on peut considérer comme les successeurs directs d'Argelander et de ses élèves; il s'est distingué, parmi ceux-là, par le soin extrême de ses innombrables observations et par la haute tenue de leurs résultats qu'il publiait avec une rare diligence.

Le Président de séance remercie le Prof. J. Hellerich, qui veut bien servir d'interprète pour la langue allemande. Il salue la présence du vénérable doyen de la Commission, le Prof. H. Ludendorff.

Sur la proposition du Président de séance, la Commission vote à l'unanimité et envoie au Comité Exécutif pour présentation à la Commission des Finances, la résolution suivante:

"Que la subvention annuelle accordée à M. Banachiewicz pour la publication des éphémérides des étoiles doubles à éclipses soit continuée à raison de huit cents francs or."

La Commission décide de publier en Appendice I du Rapport de son Président la 
Liste de roo Variables importantes dressée par M. Léon Campbell en continuation des listes de Nijland, et dont l'observation est particulièrement désirable.

Le Prof. Shapley annonce que l'Observatoire de Harvard College prépare des séquences photovisuelles de 60 champs de variables pour lesquelles il n'existe pas encore de bonnes mesures d'étoiles de comparaison. Sur la proposition de M. de Roy, celles de ces variables figurant sur la liste de Campbell seront distinguées dans celle-ci par un signe spécial.

La Commission invite celles des Associations d'observateurs d'étoiles variables qui sont en retard dans la publication de leurs observations, de l'accélérer dans toute la mesure du possible.

Le Président de séance communique que la liste d'observations encore inédites qu'il a été chargé de dresser est prête et sera incessamment publiée dans les $A$ cta Astronomica, nonobstant le fait que des séries importantes, telles que celles de Roberts, Sawyer et Yendell n'y figurent pas. De nouvelles démarches concernant ces séries seront effectuées et elles feront l'objet d'un addendum.

La Commission donne mission à son Vice-Président de centraliser et de préparer pour la publication les observations encore inédites d'étoiles variables à longue période figurant dans la liste précédente et qu'il pourra réunir.

La Commission discute ensuite point par point le Draft Report qui est adopté sans modification.

Le R. P. Stein communique qu'il espère publier en I939 la série IX de l'A.S.V., qui comprendra une majorité de longues périodes, quelques étoiles du type RV Tauri, des irrégulières, des variables de type inconnu ainsi que RS Ophiuchi. Les clichés photovisuels des champs en question seront obtenus et mesurés à Castel Gandolfo. Les magnitudes seront déterminées photoélectriquement; le Prof. Mitchell s'est offert à en faire des mesures ou des estimations visuelles selon sa méthode bien connue. Le R. P. Stein espère aussi pouvoir provoquer la publication de la suite des excellentes observations du R. P. Michael Esch, de Valkenburg, dont l'astronomie déplore la perte récente.

Au sujet de la publication du second volume du G. u. L. (Cyg à Oph), et de la deuxième liste de l'Ergänzungsheft $A . N$., la Commission tient à féliciter le Dr R. Prager du dévoûment qu'il prodigueà la communauté des chercheurs dans le domaine des étoiles variables, et forme des vœux pour la continuation de ses efforts si utiles.

La Commission recommande que tous les observateurs communiquent promptement au Prof. Kobold leurs découvertes d'étoiles variables nouvelles, dans le sens défini par la Commission de l'A.G. (\$8 du Rapport, p. 232).

Le Prof. Hertzsprung attire l'attention sur le fait qu'on publie fréquemment des séries nouvelles d'observations de variables à courte période sans l'indication précise de la meilleure époque normale qu'on pourrait en déduire et qui représenterait le jour julien moyen desdites observations; au lieu de celà, on donne une époque initiale assez arbitraire, qui sert de point zéro aux phases. Il désire indiquer cette insuffisance sérieuse, si facile à réparer.

La Commission enregistre avec une vive satisfaction la création, à l'Observatoire de Harvard College, du Bureau local Milton pour l'étude des étoiles variables, et approuve chaleureusement son programme tel qu'il lui a été exposé par Mlle Jenka Mohr. Sous la direction du Prof. Shapley et de M. et Mme Gaposchkin avec l'aide d'assistants, dont sept sont déjà à l'ouvrage, ce bureau a commencé d'étudier, sur la collection des 300,000 clichés de la Photothèque de Harvard, environ 2000 variables relativement brillantes $\left(\left\langle\mathrm{II}^{\mathrm{m}}\right)\right.$. Ce grand travail est définitivement établi pour deux ans, mais il pourra être prolongé pendant une période équivalente. 
La Commission charge son Président de séance de prendre contact avec quelques astronomes spécialisés dans l'étude spectrométrique, spectrophotométrique, dans celle des mouvements propres, parallaxes, et vitesses radiales, en vue d'examiner l'éventualité de créer en son sein une sous-commission qui favoriserait la coordination des recherches effectuées ou à effectuer dans ces divers domaines et leur liaison avec l'aspect purement photométrique de la question.

M. Cox émet le vœu que cette sous-commission s'occupe de l'ordonnance dans les recherches qu'elle tenterait de coordonner et qu'elle ne craigne pas, si la nécessité s'en faisait sentir, de suggérer une refonte complète des programmes.

La Commission considère avec faveur la détermination quantitative, par chaque observateur de variables, de son équation de couleur, et suggère que le Prof. Guthnick veuille bien établir et publier à cette fin la liste de paires d'étoiles circumpolaires dont il recommande l'observation commune.

La Commission prend acte de la déclaration du Prof. Ludendorff, que le schéma de classification générale des étoiles variables qu'il a proposé ne répond plus, sur certains points, aux faits d'observation, ainsi qu'il l'a d'ailleurs déjà constaté lui-même dans le volume supplémentaire du Handbuch der Astrophysik, et elle forme le vœu qu'il puisse, lorsque le temps en sera venu, consacrer encore sa grande expérience du problème à ce sujet important.

A la suggestion de la Commission 29, la Commission appelle l'attention des observateurs spécialisés sur l'intérêt de la détermination rapide de magnitudes visuelles ou photovisuelles des étoiles de comparaison, y compris notamment les plus faibles, pour les novae récemment découvertes.

Enfin la Commission note qu'elle n'a pas reçu de rapport concernant l'organisation d'observations collectives, sous filtre, d'étoiles variables à longue période ou irrégulières. Elle forme le vœu que les séquences spéciales indispensables pour cette organisation soient promptement constituées.

\title{
COMMISSION 28 (NEBULAE AND STAR CLUSTERS)
}

\author{
President: Prof. H. Shapley. \\ Secretary: Prof. H. L. Vanderlinden.
}

Meeting on Thursday, August 4, I938.

The report submitted by the President of the Commission was adopted.

The Chairman's proposal to appoint a sub-commission on magnitude sequences was endorsed by the Commission. This proposal was as follows:

"That the Commission on Stellar Photometry (25) consider the appointment of a sub-commission on magnitude sequences, noting that for work on stellar distribution, variable stars, external galaxies, faint asteroids and faint proper-motion stars the need of more precise photographic and visual magnitudes is very great."

With respect to this Dr Baade remarked that it was possible to transfer the magnitude scale of the observed field to the Selected Areas until $m_{\mathrm{pg}}=\mathrm{I} 7 \cdot 5$. For certain Selected Area fields, e.g. S.A. 68, photographic magnitudes have been determined down to $2 \mathrm{r} \cdot 2$. Determinations will be extended to other S.A. fields.

As to the faint galaxies, according to Messrs Shapley, Adams, Reynolds, Baade, no general classification should yet be proposed for the brighter objects and Hubble's classification is as yet sufficient. 